\title{
Elaboración y validez de un cuestionario de las habilidades motrices iniciales para estudiantes de enseñanza media chilena \\ Preparation and validation of a questionnaire on initial motor skills for students in Chilean secondary education \\ *Cristian José Oñate Navarrete, **Albert Batalla Flores, Jacqueline Del Carmen Paez Herrera \\ *Universidad Católica de Temuco (Chile), **Universidad de Barcelona (España), *** Pontificia Universidad Católica de Valparaiso
} (Chile)

Resumen. El siguiente artículo presenta la elaboración y validación de un cuestionario de habilidades motrices que mida el estado de dichas habilidades a través de la percepción de las y los profesores de Educación Física (EF) en Chile durante la transición de la enseñanza básica hacia la enseñanza media para desempeñarse correctamente en las clases de EF. Para dicha validación se ha considerado un modelo de habilidades motrices. En una primera fase se realizó el análisis y vaciado del curriìculum de Educación Física chileno de $7^{\circ}$ básico a $4^{\circ}$ año medio para luego dar paso a una segunda fase de la creación del cuestionario y posteriormente realizar la validación de contenido, de constructo y la fiabilidad del instrumento en una tercera fase a través de la recogida de datos en una muestra piloto. A partir de los datos mostrados, se concluye que el instrumento creado es válido y fiable.

Palabras Clave. habilidades motrices; evaluación motriz; test psicomotores; educación física; investigación en educación física.

\begin{abstract}
This article presents the elaboration and validation of a motor skills questionnaire that measures the state of these skills through the perception of Physical Education (PE) teachers in Chile during the transition from basic education to secondary education, so to be able to perform correctly in PE classes. For this validation, a model of motor skills has been considered. In the first phase, the analysis and emptying of the Chilean Physical Education curriculum from 7th grade to 4th year was carried out; in the second phase, the questionnaire was created and subsequently its content was validated, alongside with its construction and reliability were assessed in the third phase by collecting data from a pilot sample. From the data shown, it is concluded that the instrument created is valid and reliable. Keywords. Motor skills, motor assessment, psychomotor tests, secondary physical education.
\end{abstract}

\section{Introducción}

Las habilidades motrices en Educación Física (EF) son la base fundamental en cualquier actividad que planifique el profesor para desarrollar las competencias motoras de los y las estudiantes. Estas competencias son reconocidas y consideras un requisito mínimo para desarrollar una cultura física y deportiva a lo largo de la vida (Quitéro et al, 2017). Sin embargo, el cambio de la función actual de la educación física chilena (Doña, Jiménez, \& Gálvez, 2014; Doña \& Gálvez, 2015) al utilizar las pruebas diagnósticas para evaluar la calidad de la educación física en Chile durante la enseñanza media (SIMCE) (ACE 2013) hace que el desempeño valorado se enfoque en la medición de funciones motoras alejadas del contexto educativo (Scheuer, Herrmann \& Bund, 2019) sin centrarse en un diagnóstico previo en cómo se adquieren y desarrollan las habilidades motrices en la transición de la enseñanza básica a la enseñanza media. Proceso necesario para generar y consolidar los hábitos saludables de una práctica autónoma y continuada de actividad física (AF) en la vida del adolescente (de Mesa, Estrada, Prado \& González, 2009).

El actual escenario escolar hace que la intervención en EF pierda su enfoque central de experimentar la motricidad y la diversificación de los estímulos. Esto genera que la EF se transforme en una mejora de la capacidad física que favorece los indicadores de calidad docente y no hacia la adquisición de las habilidades orientadas hacia una respuesta motora de calidad (Scheuer et al., 2019). Chile además tiene otro escenario complejo para la EF puesto que en los últimos años las

Fecha recepción: 08-11-19. Fecha de aceptación: 01-03-20

Cristian José Oñate Navarrete

kinecristianjose@gmail.com tasas de obesidad han ido en aumento. Según los últimos datos publicados la OCDE (2019) Chile se posiciona como líder internacional en obesidad tanto en adultos como en niños. Esto hace que el escenario escolar al que se enfrenta el profesorado difiera mucho al de décadas anteriores. Strodden (2008) citado en Quitéro et al (2017) señala que en las últimas décadas se ha prestado menos atención al desarrollo de las competencias motoras esenciales para el desarrollo de la actividad física.

Aunque existen diversos test motores para evaluar las habilidades motrices, parte de los investigadores se enfoca en etapas tempranas de la infancia centrándose solo en las habilidades motrices básicas (HMB), sin embargo, no plantean una etapa intermedia de aprendizaje necesaria para adquirirlas y conectar el desarrollo hacia las habilidades motrices especializadas (HME). En este contexto los instrumentos que miden las habilidades motrices intermedias (HMI) entre las HMB y las HME y la transición de estas más allá de los 12 años de edad en contextos escolares son casi nulos. Por tanto, surge la necesidad de crear un instrumento válido (cuestionario), que considere pedagógicamente una etapa de adquisición de HMI para completar el vacío teórico existente actualmente en el modelo de clasificación de habilidades motrices de Castañer \& Camerino (2006) y en los instrumentos de medición motrices utilizados por diversos autores en edades comprendidas entre los 12 y 18 años (Caminero, 2002; Gómez, 2004; Ruiz-Pérez, Barriopedro-Moro, Ramón-Otero, Palomo-Nieto, Rioja-Collado, García-Coll \& NaviaManzano, 2017; Mesa, Estrada, Prado \& González, 2009; Scheuer, Herrmann \& Bund, 2019).

Por otro lado, existe evidencia actual de la influencia positiva del desarrollo de las habilidades motrices en EF a lo largo de la escolarización tanto para el desarrollo de capacidades cognitivas como para la adherencia a la AF en la ado- 
lescencia (Callcot, Hammond \& Hill, 2015; Wu, Lian, Lu \& Wang, 2017; Robinson et al., 2016 citados en García-Marín \& Fernández-López, 2020; Zueck et al, 2020). De esta manera parece interesante el conocer en una primera instancia cuáles son las HMI más relevantes utilizadas en el contexto escolar de la enseñanza media, considerando la propia opinión de los docentes de EF. Sin embargo, a la fecha no existen estudios de este tipo. Por lo tanto, el objetivo de esta investigación es diseñar y validar un instrumento denominado cuestionario de habilidades motrices intermedias para estudiantes que ingresan a la enseñanza media chilena (HMIEMCHI) dejando en claro que esta investigación no es una metodología de trabajo sobre las prácticas docentes, sino más bien es un aporte metodológico para la enseñanza en EF que busca aportar al estado del arte a través de la generación de un instrumento que considera el proceso de adquisición de las HMI durante la escolarización de los y las estudiantes chilenas, el cual puede ser considerado como un insumo para futuras investigaciones y creaciones de test motrices contextualizados a la realidad escolar nacional en una etapa adolescente. Para la construcción del cuestionario nos basaremos tanto en el planteamiento de clasificación de habilidades motrices de Batalla (2000) como en el análisis y vaciado del currículum chileno.

\section{Material y Método}

Desde el punto de vista práctico, debido a que el interés de investigación está centrado en conocer una realidad específica de la EF en Chile, el enfoque cuantitativo, favorece la recolección y sistematización de la información arrojada por el cuestionario (Hernández, Fernández \& Baptista, 2006). Así, para esta investigación, es prudente hacer una primera aproximación a la importancia asignada a las habilidades motrices desarrolladas en los y las adolescentes de la enseñanza media, pues el estado del arte del contexto al que se quiere aproximar carece de información. Se ha optado por el diseño de investigación no experimental cuantitativo ya que a través de su lógica permite generalizar más ampliamente los resultados (Hernández et al., 2006). El tipo de diseño fue transeccional descriptivo ya que se recolectaron datos en un tiempo único con el fin de describir los fenómenos y analizar su incidencia en un momento dado (Hernández et al., 2006).

\section{Características del cuestionario}

El objetivo de esta investigación es diseñar y validar un cuestionario que recoja el grado de importancia de las habilidades motrices intermedias para estudiantes que ingresan a la enseñanza media chilena (HMI-EMCHI). Por ello, la calidad del cuestionario recae básicamente en la clase de preguntas formuladas y en su adecuada formulación, siendo esta la expresión en forma interrogativa de las variables de las cuales se requiere información (Murillo, 2017). Para esto se identificó la primera variable, presencia de las habilidades motrices en el curriìculum de EF chileno de $7^{\circ}$ básico a $4^{\circ}$ año medio, para verificar que tan próximo está el curriìculum de EF a la enseñanza de las habilidades motrices bajo el modelo de clasificación expuesto por Batalla (2000).

Primero se comparó la cantidad de habilidades motrices
(HM) presentes en el curriìculum de EF con el modelo expuesto de Batalla (2000) en donde se realizó una planilla Excel con un listado de $36 \mathrm{HM}$ y sus respectivas definiciones propuestas por el autor Batalla (2000) las cuales fueron cotejadas con el vaciado del currículum chileno. Para esto se analizaron ocho fuentes de información que, por su naturaleza, abordan el tema (MINEDUC, 2004; 2009; 2015;2016) en donde se evidenció que la enseñanza de las HM en el curriìculum se da principalmente en cuatro dimensiones: deportes individuales sin oposición (carrera, prueba de salto, gimnasia artística, escalada, etc.) deportes individuales con oposición (tenis, bádminton, esgrima, etc.), deportes colaborativos sin oposición (kayak en pareja, prueba de relevos, etc.) y deportes colaborativos con oposición (basquetbol, hándbol, futbol, etc.). Con esta información se logró analizar los diferentes deportes expuestos en él curriìculum y las HM que se utilizaban para su ejecución. Seguidamente se llevó esta información a la planilla, clasificándola en las categorías expuestas por Batalla (2000) correspondientes (a) desplazamiento, (b) salto, (c) giro, (d) manejo y control de objetos con las manos, (e) manejo y control de objetos con los pies, la cabeza u otro objeto, (f) deslizamiento, $(\mathrm{g})$ transporte y $(\mathrm{h})$ habilidades acuáticas. De las cuales se disgregan solo 23 habilidades motrices aplicadas al contexto escolar de las 36 expuestas por el autor Batalla (2000) y que se trabajaban en los deportes presentes de los ocho documentos del MINEDUC mencionados en la figura 1.

Para recoger la opinión del profesorado de EF, la segunda variable denominada fue el grado de importancia que ellos le atribuyen a este tipo de HM en el ámbito escolar, para lo cual se expresó la valoración de las respuestas a través de una escala de Likert de uno al cinco según el grado de acuerdo con la misma. Siendo (1) no es importante, (2) poco importante, (3) imparcial, considerando este punto como una respuesta necesaria para representar las opiniones neutrales con el fin de no forzar las respuestas emitidas, (4) importante, (5) muy importante. Se ha escogido esta escala por que los expertos recomiendan usar respuestas graduadas siempre que sea posible, para favorecer el análisis de las respuestas con una escala de Likert de al menos 5 categorías (Ferrando \& Anguiano-Carrasco 2010; Frías-Navarro \& Soler, 2013). Un factor a tener en cuenta para el número de preguntas es el tamaño de la muestra y la cercanía a esta (Murillo, 2017). Por tal motivo se escogió un número de 23 preguntas que reflejan el análisis de la primera variable, aplicadas a una muestra escogida por conveniencia a estudiantes universitarios $(N=42)$ de último nivel de EF ( $71 \%$ son hombres y $29 \%$ son mujeres) pertenecientes a la Pontificie Universidad Católica de Valparaíso, siendo la Dra. Jacqueline Paéz, coordinadora académica de postgrado de la Escuela de EF, la académica responsable de la aplicación del cuestionario, en formato impreso, bajo un contexto de participación voluntaria de los estudiantes, sin recibir recompensa alguna.

\section{Decidir el tipo, formulación y orden de las preguntas}

Al decidir el tipo de preguntas se optó por una batería de preguntas cerradas y politómicas a excepción de la última que fue una pregunta abierta para permitir la respuesta libre al considerar otra habilidad motriz no mencionada en el cues- 
tionario, sin embargo, esta no fue considerada en el análisis estadístico final del estudio. En cuanto a la formulación del enunciado de las preguntas fue formulada de tal manera que no ejerciera influencia en las respuestas, procurando un diseño formal, donde la primera página expone la explicación y las instrucciones para su cumplimiento y en las páginas siguientes se ordenaron las preguntas por las categorías de desplazamiento, giro, etc. situando juntas las preguntas de una misma categoría, por ejemplo, categoría de salto se ordenaron todas las preguntas de salto de distancia, salto estético y salto con manipulación de objetos, como se observa en la tabla 1 de resultados.

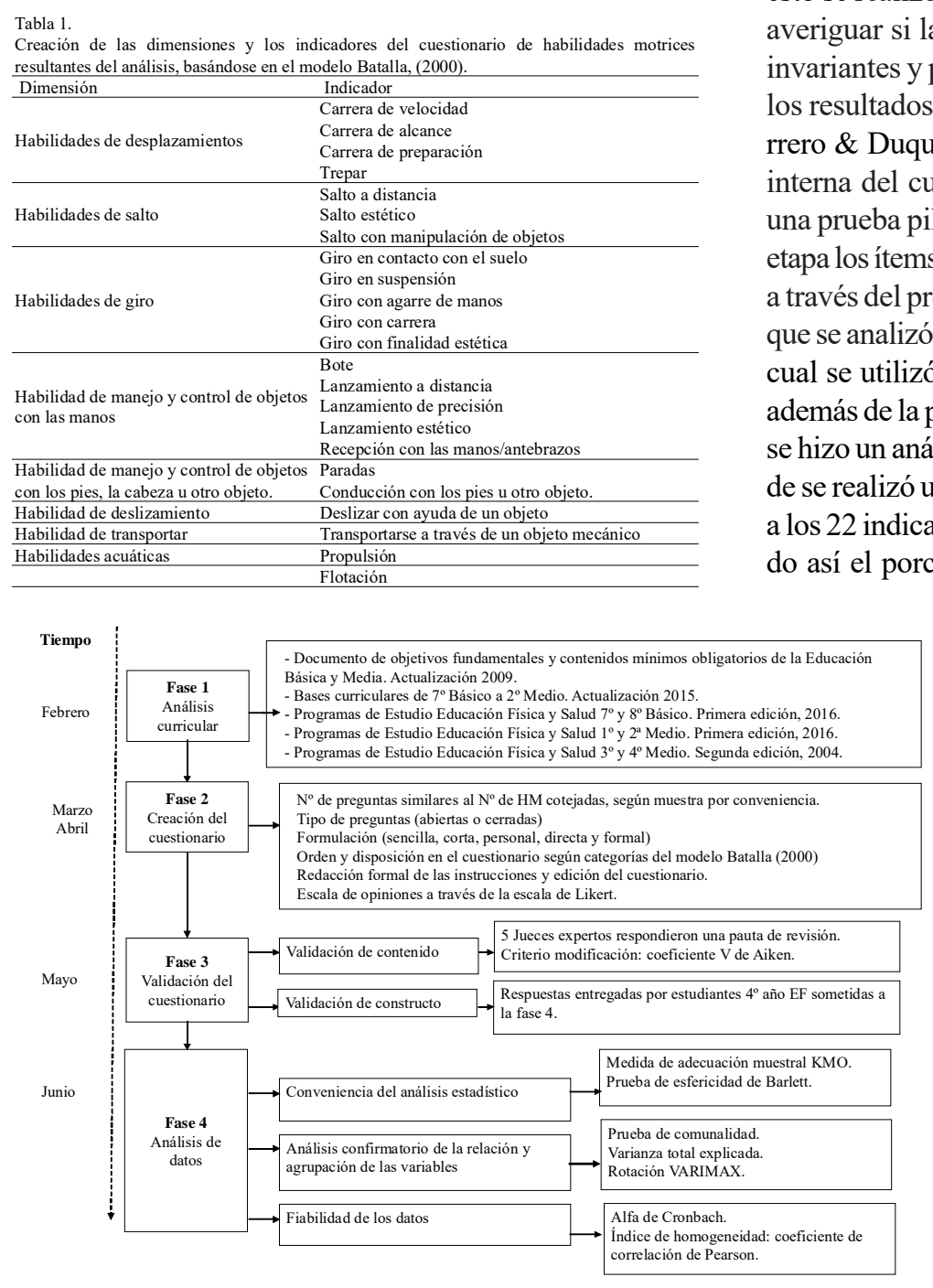

Figura 1. Proceso metodológico llevado a cabo para la construcción del cuestionario de Habilidades Motrices intermedias para la enseñanza media.

\section{Validación del cuestionario}

La validez alude a la capacidad del instrumento de medir el constructo que pretende cuantificar (Lamprea, GoìmezRestrepo, 2007 citado en Campo-Arias \& Oviedo, 2008). En esta etapa se procedió a la validación del cuestionario basado en dos estrategias: la validación de contenido y validación de constructo (Murillo, 2017).

Para determinar la validez de contenido y el grado de comprensión de las preguntas se elaboró una pauta de revisión crítica del cuestionario para evaluar la pertinencia y la claridad conceptual de las dimensiones y sus ítems al propó- sito del cuestionario, así también un espacio para proponer cambios y/o sugerencias. Para esto se convocó a dos doctores de España y tres de Chile con un perfil enfocado al área de desarrollo motriz o deportivo, contar con el grado de doctor, ser docente universitario y ser profesor de Educación Física de formación a quienes se les solicitó responder el cuestionario para hacer una validación de contenido con la finalidad de ver el grado de correspondencia entre el enunciado del ítem y lo que se pretendía medir, velar por la sintaxis y la comprobación de la coherencia y claridad conceptual del cuestionario (Murillo, 2017).

En cuanto a la validación de constructo del cuestionario esto se realizó con la finalidad de explorar el cuestionario y averiguar si las relaciones entre las variables se mantienen invariantes y pueden servir de base para la interpretación de los resultados en distintas poblaciones (Rodero, Díaz, Gue\& Duque, 2016). Para esto se analizó la consistencia interna del cuestionario y su fiabilidad, llevándose a cabo una prueba piloto con la muestra antes mencionada. En esta etapa los ítems del instrumento se analizaron estadísticamente a través del programa IBM SPSS Statistics 25.0. Lo primero que se analizó fue si el modelo factorial era adecuado, para lo utilizó la prueba de Kaiser, Meyer y Olkin (KMO) además de la prueba de esfericidad de Barlett. Seguidamente se hizo un análisis de la validez interna del cuestionario donde se realizó una prueba de comunalidad para luego someter a los 22 indicadores a una varianza total explicada conociendo así el porcentaje de representatividad de las preguntas. Luego se realizó una rotación VARIMAX usando una carga factorial de 0.7-0.8 la cual arrojó a diez habilidades motrices como las más significativas. Para finalizar y evaluar la consistencia interna del cuestionario, se utilizó un Alfa de Cronbach (Macías, 2007) y el índice de homogeneidad (Rodero et al., 2016). Este proceso metodológico llevado a cabo se resume esquemáticamente en la figura 1.

\section{Resultados}

En este apartado daremos a conocer los principales hallazgos resultantes del trabajo investigativo en tres fases.

Primera fase: análisis y vaciado del currículum chileno para detectar la presencia de las diferentes habilidades motrices

Esta investigación se basa en un contexto puntual de la educación física chilena, pues se analizó el currículum educativo de $7^{\circ}$ básico a $4^{\circ}$ año medio. Se concuerda con estudios similares que analizan los planes y programas de estudios de la educación física (Moreno, Jiménez \& Gálvez, 2014) al visualizar que si bien existen cambios en el curriìculum a lo largo del tiempo este aún se percibe con un excesivo énfasis en la enseñanza de los deportes sin atender a las necesidades actuales del país. Además, la presencia temática en los programas curriculares de enseñanza media sobre habilidades motrices y rendimiento motor apenas alcanza el 10\% 
hasta el año 2015 según el análisis cuantitativo expuesto por Moreno et al. (2014) existiendo un desarrollo carente en este ámbito. Otro punto importante a destacar de este análisis es la desactualización de los programas de $3^{\circ}$ y $4^{\circ}$ medio (2004) en los cuales se cita la ampliación y profundización de las HM adquiridas en niveles inferiores. Sin embargo, esta cita hace referencia en su entonces a una profundización al antiguo curriìculum de $7^{\circ}$ y $2^{\circ}$ medio, pues actualmente se observa una desconexión de la estructuración del programa curricular del 2004, basado en objetivos, en relación a los actuales programas de $7^{\circ}$ a 2 medio del año 2015 que basan el contenido por competencias.

Ahora bien, de los tres ejes temáticos del programa de educación física y salud de $7^{\circ}$ y $8^{\circ}$ Básico solo se consideró el eje temático de habilidades motrices, relevante para este trabajo, el cual busca en este nivel seleccionar, combinar y aplicar estas habilidades para deportes individuales, de oposición, colaboración y oposición/colaboración, siendo las habilidades motrices con mayor presencia las de manipulación, locomoción y estabilidad (MINEDUC 2016). El programa de estudio de $1^{\circ}$ y $2^{\circ}$ Medio trabaja las habilidades motrices con un mayor control y precisión que en los niveles anteriores. Aquí se observa que las habilidades motrices tributan hacia la especificación deportiva sobre todo en $2^{\circ}$ año medio (MINEDUC, 2016). El programa de $3^{\mathrm{a}}$ y $4^{\mathrm{a}}$ año medio a diferencia de los anteriores fue considerado para este análisis solo el ámbito de aptitud física y motriz asociada a salud y calidad de vida (MINEDUC, 2004).

\section{Segunda fase: Creación del cuestionario.}

Para efectos de la creación de este cuestionario fueron seleccionadas 23 de las 32 habilidades motrices expuestas en el modelo de Batalla (2000) que son abordadas claramente en el currículum, dejando de lado habilidades motrices tales como la reptación, la cuadrupedia o los saltos y giros en medio acuático por mencionar algunos debido a que se integran en otras habilidades ya seleccionadas. A continuación, en la tabla 1 se exponen la creación de las dimensiones y los indicadores del cuestionario que se trabajan en el curriìculum chileno resultantes del análisis bajo el modelo de Batalla (2000).

\section{Tercera fase: Validación del cuestionario.}

El diseño de las preguntas del cuestionario fue expuestas a una validación de contenido la cual no suele ser expresada cuantitativamente a través de un índice o coeficiente, sino que, por lo general, se estima de manera subjetiva a través de juicio de expertos (Rodero, et al., 2016). En este análisis hubo una valoración cualitativa de la redacción y coherencia las cuales fueron transformadas en variables cuantitativas para objetivar el resultado utilizando el coeficiente de validez $\mathrm{V}$ de Aiken como criterio de modificación siendo estadísticamente significativo un coeficiente superior o igual a 0,5 (Mayaute, 1988).

Una vez realizada las modificaciones solicitadas por los expertos se procedió a realizar la validación del constructo del cuestionario, la cual se llevó a cabo en una prueba piloto a través de un muestreo por conveniencia a la unidad de análisis conformada por los y las estudiantes de Educación Física de la Pontificia Universidad Católica de Valparaíso
$(N=42)$. Los y las estudiantes encuestadas son de $4^{\circ}$ año de EF que tienen en promedio 22 años. En esta prueba piloto participaron 37 estudiantes, a quienes les fue autoadministrado el instrumento en cuestión, sin embargo, la pregunta asociada al indicador de lanzamiento a distancia no fue respondida por error durante la impresión del instrumento. A continuación, en la tabla 2 se puede encontrar el cuestionario completo con las modificaciones post validación de expertos. En cuanto al análisis de la validez de constructo se sometieron las respuestas de los y las estudiantes de EF chilenos a un análisis estadístico expuesta en la cuarta fase.

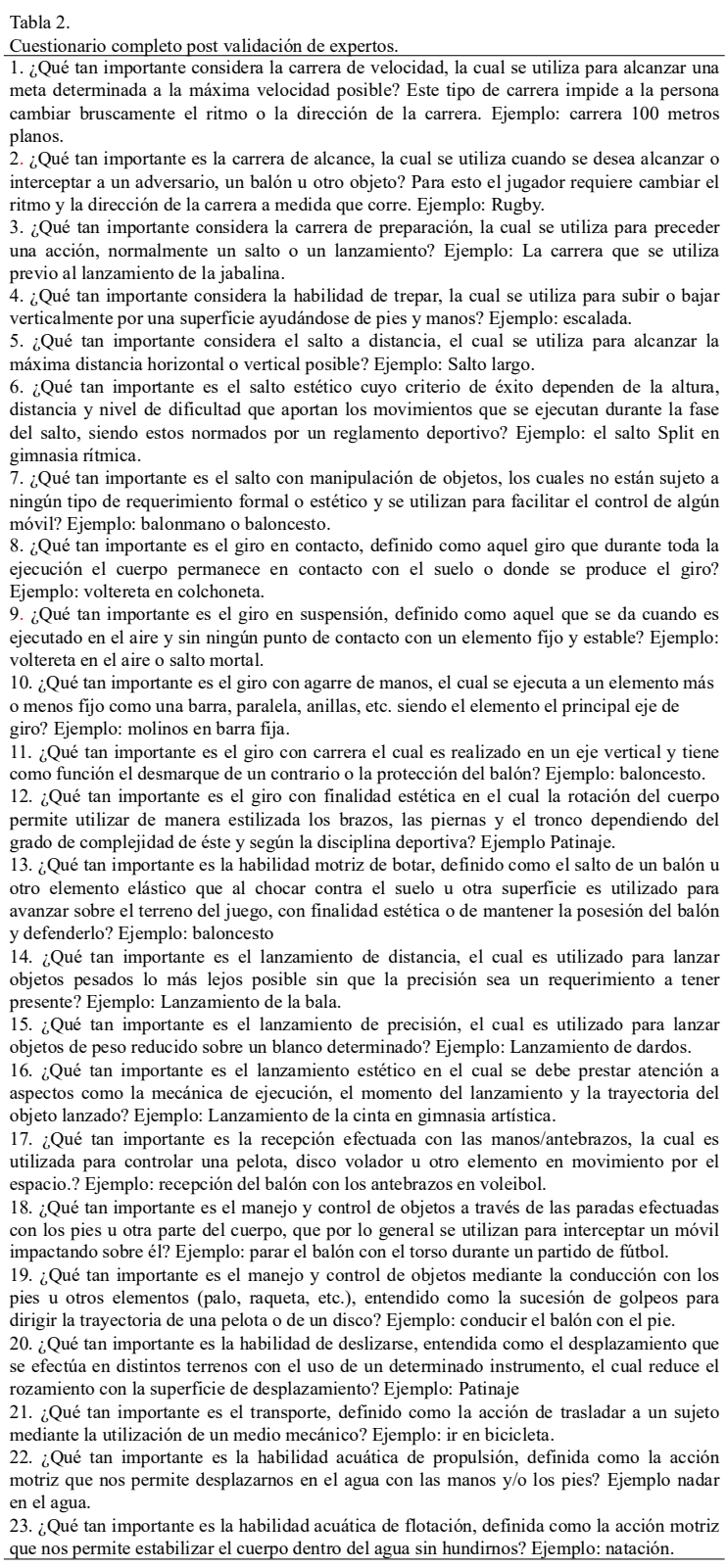

22. ¿Qué tan importante es la habilidad acuática de propulsión, definida como la acción motriz que nos permite desplazarnos en el agua con las manos y/o los pies? Ejemplo nadar en el agua.

23. ¿Qué tan importante es la habilidad acuática de flotación, definida como la acción motriz que nos permite estabilizar el cuerpo dentro del agua sin hundirnos? Ejemplo: natación.

\section{Cuarta Fase: Análisis de datos}

Para analizar la adecuación de la muestra y la conveniencia del análisis estadístico se aplicó un análisis factorial de adecuación muestral de Kaiser-Meyer-Olkin (KMO) y una prueba de esfericidad de Barlett arrojando un 0,611 y un 0.0 respectivamente. Respecto al análisis confirmatorio de la relación y agrupación de las variables se realizó una prueba de comunalidad de cada pregunta para probar la validez interna 
del cuestionario, obteniendo un valor en su extracción $>0.4$. En cuanto a la varianza total explicada como se observa en la tabla 3, únicamente los siete primeros factores tienen valores propios mayores que 1 y explican el $72,5 \%$ de la varianza, esto quiere decir que con estos 7 factores se puede representar el 72,5\% del problema de investigación. Dicho de otra manera, sólo son relevantes 7 factores para resumir las variables originales del problema (Súares, 2007).

No obstante, la poca claridad en la carga factorial de las variables mostradas por la matriz, resulta necesario efectuar una rotación ortogonal que permita reducir ambigüedades en las cargas factoriales de las variables y hallar una solución más clara. Para esto se utilizó el método de rotación VARIMAX que busca redistribuir la varianza a lo largo de todos los componentes en la matriz de carga (Suárez, 2007). De este modo se han reducido las 22 variables a solo 10 elementos para la construcción de la tabla 4 que expresa la matriz de componentes rotados con puntuaciones factoriales entre 0.8 y 0.7 de la rotación VARIMAX.

En el análisis de la consistencia interna y la fiabilidad de los datos, las 22 preguntas respondidas del cuestionario, se obtuvo un coeficiente de Alfa de Cronbach de 0.872 , el cual excede el valor mínimo de 0,70 para determinar la confiabilidad de la prueba (Macías, 2007).

$\mathrm{Al}$ analizar el índice de homogeneidad de los ítems que componen el cuestionario con el coeficiente de correlación de Pearson para conocer el grado en que dicho ítem está midiendo lo mismo que la prueba globalmente; es decir, del grado en que contribuye a la consistencia interna del test (Rodero et al., 2016) este arrojó un índice mayor a 0.2.

\begin{tabular}{|c|c|c|c|c|c|c|c|c|}
\hline \multirow[b]{3}{*}{ Componente } & \multicolumn{2}{|c|}{ Autovalores iniciales } & \multicolumn{3}{|c|}{$\begin{array}{l}\text { Sumas de cargas al } \\
\text { cuadrado de la extracción }\end{array}$} & \multicolumn{3}{|c|}{$\begin{array}{l}\text { Sumas de cargas al cuadrad } \\
\text { de la rotación }\end{array}$} \\
\hline & $\% \mathrm{de}$ & $\%$ & & $\% \mathrm{de}$ & $\%$ & & $\% \mathrm{de}$ & $\%$ \\
\hline & Total varianza & acumulado & Total & varianze & acumulado & o Total & varianza & acumulado \\
\hline 1 & $6,415 \quad 29,159$ & 29,159 & 6,415 & 29,159 & 29,159 & 2,831 & 12,868 & 12,868 \\
\hline 2 & $2,775 \quad 12,614$ & 41,773 & 2,775 & 12,614 & 41,773 & 2,748 & 12,490 & 25,358 \\
\hline 3 & $1,602 \quad 7,283$ & 49,057 & 1,602 & 7,283 & 49,057 & 2,564 & 11,656 & 37,014 \\
\hline 4 & $1,469 \quad 6,676$ & 55,733 & 1,469 & 6,676 & 55,733 & 2,414 & 10,971 & 47,986 \\
\hline 5 & $1,435 \quad 6,522$ & 62,255 & 1,435 & 6,522 & 62,255 & 2,089 & 9,495 & 57,481 \\
\hline 6 & $1,200 \quad 5,455$ & 67,709 & 1,200 & 5,455 & 67,709 & 1,856 & 8,438 & 65,918 \\
\hline 7 & $1,066 \quad 4,847$ & 72,556 & 1,066 & 4,847 & 72,556 & 1,460 & 6,638 & 72,556 \\
\hline \multicolumn{9}{|c|}{$\begin{array}{l}\text { Tabla } 4 . \\
\text { Componentes resultantes }\end{array}$} \\
\hline \multicolumn{9}{|l|}{ Componente } \\
\hline & \multirow{2}{*}{\multicolumn{8}{|c|}{$\begin{array}{l}\text { Salto con manipulación de objetos y deslizamientos } \\
\text { Giro en suspensión }\end{array}$}} \\
\hline Y2 & & & & & & & & \\
\hline Y3 & \multicolumn{8}{|c|}{ Carrera de velocidad y trepar } \\
\hline Y4 & \multicolumn{8}{|c|}{ Carrera de alcance } \\
\hline Y5 & \multirow{2}{*}{\multicolumn{8}{|c|}{ Propulsión y flotación }} \\
\hline Y6 & \multirow{2}{*}{\multicolumn{7}{|c|}{ Conducción con los pies u otros objetos }} & \\
\hline Y7 & & & & & & & & \\
\hline
\end{tabular}

\section{Discusión de resultados}

El objetivo de esta investigación fue diseñar y validar un cuestionario de HMI-EMCHI, en donde el análisis estadístico que se llevó a cabo para su construcción arrojó que el instrumento es válidos y fiables ya que en el diseño y validación fue analizado rigurosamente por los cinco expertos y conocedores del tema, obteniendo una validez de contenido superior o igual 0.5 a través del coeficiente $\mathrm{V}$ de Aiken. Las preguntas consideradas en la valoración de los y las expertas fueron aquellos que tenían acuerdo de opinión de al menos tres de ellos. Respondiendo así a la pregunta ¿cuán pertinente es la pregunta para el cuestionario? los resultados obtenidos, muestran una pertinencia de un $70 \%$ de las preguntas para el cuestionario total, arrojando solo un $30 \%$ de no pertinencia compuesto por las preguntas 1,3,4,9,10,12,14. Respecto a la pregunta ¿la redacción es clara y comprensible? en las preguntas $1,2,6,12,17,18,19,21$ son aquellas que fueron reestructurada en su redacción pues solo hubo acuerdo en dos de los cinco expertos (as). Para el caso ¿la pregunta favorece doble interpretaciones o ambigüedades en las respuestas? fueron consideradas las preguntas $1,2,5,6,12,14,16,17,19$ para reestructurar la claridad de los ejemplos.

Respecto a la validación de constructo los resultados nos muestran que la muestra es estable y posee una buena adecuación a los datos ya que el análisis factorial KMO arrojo un 0,611 con un 0.0 de esfericidad de Barlett respectivamente. En cuanto al análisis de la comunalidad de cada pregunta para probar la validez interna del cuestionario se obtuvo un resultado en su extracción $>0.4$, por tanto, se concluye que existe asociación y coherencia de un ítem con otro en la estructura del cuestionario. Referente a la consistencia interna de las 22 preguntas se obtuvo un Alfa de Cronbach de 0.872 , por esta razón, se puede establecer que existe una buena correlación entre los reactivos del instrumento y una adecuada consistencia interna del instrumento diseñado lo cual es corroborado por el análisis de homogeneidad donde el coeficiente de Pearson mayor a 0.2 establece que los 22 ítems obtenidos miden y representan efectivamente la dimensión a la cual corresponde cada uno.

La mayor limitante de esta investigación es que el instrumento diseñado al circunscribirse bajo un contexto netamente educativo de la asignatura de $\mathrm{EF}$, es poco extrapolable a otras áreas de estudio, pues para este análisis solo se consideró los niveles de $7^{\circ}$ básico a $4^{\circ}$ año medio, por lo tanto, la generalización de los datos estará enfocado bajo este contexto. Además, otro punto a considerar es la elección de las habilidades motrices del cuestionario que fueron recopiladas de los programas de EF en donde se observa que el programa de $3^{\circ}$ y $4^{\circ}$ medio presentes en la página del MINEDUC actualmente son del año 2004, por tanto, se recomienda que para futuras investigaciones en el área sean reevaluadas las HM de estos niveles.

Para finalizar, este trabajo propone un instrumento que pueda aportar al área de la $\mathrm{EF}$, entregando un cuestionario válido y fiable, construido a partir de la evidencia científica y contextualizado al ámbito nacional. Otra contribución importante es que el instrumento diseñado es capaz de hacer un levantamiento de la información que considera la opinión docente, permitiendo tener un mayor conocimiento respecto a cuáles son las habilidades motrices que se requieren para tener un correcto desempeño en clases de EF, puesto que la perspectiva predominante en los cuestionarios y test motrices analizados, realizan un levantamiento de la información aislada del contexto educativo nacional y no consideran a los docentes involucrados en el ejercicio de la profesión, limitándose a un escaso número de habilidades motrices, que muchas veces tienen un enfoque de aplicación diagnóstica o de monitoreo de capacidades motoras estándar con parámetros de otros contextos sociales, centrando sus esfuerzos solo en aprendizajes motrices básicos. Sin embargo, para efectos de este estudio se postuló que los aprendizajes motrices están centrados en aprendizajes de habilidades intermedias para cada actividad física y deportes que se 
explicitan en los programas de $7^{\circ}$ básico a $4^{\circ}$ año medio de la EF chilena, concordando con autores como Magill (1993) citado en Suárez \& Hernández (2007) y Batalla (2000) quienes señalan que se aprende lo que se práctica pero nunca lo que no se práctica, llamando a esto transferencia motriz. Podemos decir por tanto que lo enseñado en clases será transferible a aquellos entornos que tengan una mayor similitud con las HM explicitadas en los programas de estudio, expresando que es necesario el aporte que hace este estudio al entregar un instrumento que nos permita recopilar la información que nos entregan los docentes EF, para saber que necesitan los y las adolescentes a la hora de enfrentarse a las clases de EF.

\section{Conclusiones y aplicaciones.}

Al haber elaborado y validado un cuestionario de habilidades motrices intermedias para estudiantes de enseñanza media chilena (HMI-EMCHI) se propone con este instrumento la posibilidad de tener nuevas herramientas de investigación en el ámbito de la EF para que los profesores tematicen acerca de las habilidades motrices que requieren mayor importancia durante la enseñanza de la EF chilena contextualizada a la realidad escolar donde sea aplicado el instrumento.

Se debe señalar que este instrumento metodológicamente posee una buena validez de contenido y una validez de constructo puesto que el análisis factorial arrojó siete factores que explican el 72,5\% de la varianza total lo que generó un cuestionario acotado a diez habilidades motrices que fueron consideradas como importantes por la muestra piloto, siendo igual de válido que la versión del instrumento extendida la que contiene 22 habilidades motrices sometidas a análisis. Además, los resultados de la medida de adecuación muestral KMO y la prueba de Barlett establecen que desde el punto de vista de su constructo este cuestionario de 22 indicadores es válido para evaluar la opinión docente de las habilidades motrices que poseen mayor relevancia.

A partir de los resultados anteriormente expuestos, se puede determinar que este cuestionario es válido y pertinente tanto para 10 reactivos con mayor relevancia en los componentes de salto con manipulación de objetos y deslizamientos, giro en suspensión, carrera de velocidad y trepar, carrera de alcance, propulsión y flotación, conducción con los pies u otros objetos y recepción con las manos o antebrazos que reflejó una buena consistencia interna, pues esta alcanzó un alfa de Cronbach de 0,72. La totalidad del instrumento, con $22 \mathrm{HM}$ en tanto, arrojó un índice de 0,87 lo cual demuestra un alto índice de fiabilidad.

En este sentido este trabajo invita a futuras investigaciones a utilizar el instrumento y a reflexionar sobre cuál es el grado de importancia de las habilidades motrices intermedias que le otorgan los y las docentes en EF bajo contextos particulares del quehacer trayendo consigo importante información que contribuya al desarrollo del proceso educativo en el contexto escolar. Además, se sugiere que para evitar los costes económicos y los errores de impresión del cuestionario la distribución y aplicación se realicé de manera digital ampliándose a un número mayor de profesores en las distintas regiones para considerar diversos contextos educativos.

\section{Agradecimientos}

Agradecemos a la Comisión Nacional de Investigación Científica y Tecnológica de Chile (CONICYT) por haber hecho posible esta investigación a través de la Beca de Magister en el extranjero Becas Chile, convocatoria 2018.

\section{Referencias}

ACE. (2013). Sistema de medición de la calidad de la educación. Santiago de Chile: Gobierno de Chile.

Batalla, A., (2000). Habilidades motrices, Barcelona, España, editorial INDE publicaciones.

Callcott, D., Hammond, L., \& Hill, S. (2015). The Synergistic Effect of Teaching a Combined Explicit Movement and Phonological Awareness Program to Preschool Aged Students. Early Childhood Education Program, 43(3), 201211.

Caminero, F. (2002). Diseño y estudio científico para la validación de un test motor original, que mida la coordinación motriz en alumnos/as de educación secundaria obligatoria. https://doi.org/10.3163/1536-5050.99.3.008

Campo-Arias, A., \& Oviedo, H. (2008). Propiedades psicométricas de una escala: la consistencia interna. Revista de salud pública, 10, 831-839.

Castañer, M. \& Camerino, O. (2006). Manifestaciones básicas de la motricidad. Universitat de Lleida.

De Mesa, C., Estrada, J., Prado, J. \& González, C. (2009). Disponibilidad de las habilidades motrices en escolares de 4 a 14 años: Aplicabilidad del test de Desarrollo Motor Grueso de Ulrich. Aula abierta, 37(2), 19-28

Doña,A. \& Gálvez, C. (2015). La educación física chilena y su profesorado: proponiendo algunos retos para la investigación en el área. Retos: nuevas tendencias en educación física, deporte y recreación, (28), 291-296.

Doña,A., Jiménez, R., \& Gálvez, C. (2014). La educación física en Chile: análisis crítico de la documentación ministerial. Revista Brasileira de Ciências do Esporte, 36(2), 411-427.

Ferrando, P., \& Anguiano-Carrasco, C. (2010). El análisis factorial como técnica de investigación en psicología. Papeles del psicólogo, 31(1), 18-33.

Frías-Navarro, D., \& Soler, M. (2013). Prácticas del análisis factorial exploratorio (AFE) en la investigación sobre conducta del consumidor y marketing. Artículos en PDF disponibles desde 1994 hasta 2013. A partir de 2014 visítenos en www. elsevier. es/sumapsicol, 19(1).

García-Marín, P., \& Fernández-López, N. (2020). Asociación de la competencia en las habilidades motrices básicas con las actividades físico- deportivas extracurriculares y el índice de masa corporal en preescolares Association of the fundamental movement skills competence with the extracurricular sports and the body mass index in preschoolers, 2041, 33-39

Gómez, M. (2004). Problemas evolutivos de coordinación motriz y percepción de competencia en el alumnado de primer curso de educación secundaria obligatoria en la clase de educación física (Doctoral dissertation, Universidad Complutense de Madrid, Servicio de Publicaciones). 
Hernández, R., Fernández, C., \& Baptista, P. (2006). Metodología de la investigación (Vol. 4). México.

Macías, A. (2007). ¿Cómo valorar un coeficiente de confiabilidad? Investigación educativa duranguense, (6), 6-10.

Mayaute, L. (1988). Cuantificación de la validez de contenido por criterio de jueces. Revista de psicología, 6(1-2), 103-111.

MINEDUC, Bases curriculares de $7^{\circ}$ a $2^{\circ}$ medio. Currículum Nacional. Actualizado (2015). Recuperado de https:// www.curriculumnacional.cl/614/articles-37136_bases.pdf

MINEDUC, Marco Curricular. Objetivos fundamentales y contenidos mínimos obligatorios de la educación básica y media. 2009. Recuperado de https:// www.agenciaeducacion.cl/wp-content/uploads/2013/02/ Marco-Curricular-y-Actualizacion-2009-I-a-IV-Medio.pdf

MINEDUC, Programa de estudio de Educación Física, Cuarto Año Medio, Formación General. Segunda ed., (2004). Recuperado de https://www.curriculumnacional.cl/614/ articles-34439 programa.pdf

MINEDUC, Programa de estudio de Educación Física, Octavo Año Básico, Formación General. Primera ed., (2016). Recuperado de https://www.curriculumnacional.cl/614/ articles-20745 programa.pdf

MINEDUC, Programa de estudio de Educación Física, Primer Año Medio, Formación General. Primera ed., (2016). https://www.curriculumnacional.cl/614/articles34436_programa.pdf

MINEDUC, Programa de estudio de Educación Física, Segundo Año Medio, Formación General. Primera ed., (2016). Recuperado de https:// www.curriculumnacional.cl/614/articles34437 programa.pdf

MINEDUC, Programa de estudio de Educación Física, Séptimo Año Básico, Formación General. Primera ed., (2016). Recuperado de https://www.curriculumnacional.cl/614/ articles-20744_programa.pdf

MINEDUC, Programa de estudio de Educación Física, Tercer Año Medio, Formación General. Segunda ed., (2004).
Recuperado de https://www.curriculumnacional.cl/614/ articles-34438 programa.pdf

Murillo, F. (2017). Cuestionario y escalas de actitudes.

OCDE (2019). Estudios de la OCDE sobre salud pública, Chile hacia un futuro más sano, Ministerio de Salud, Gobierno de Chile 2019.

Pastor, V, Brunicardi, D., Arribas, J., \& Aguado, R. (2016). Los retos de la Educación Física en el Siglo XXI. Retos. Nuevas tendencias en Educación Física, Deporte y Recreación, (29), 182-187.

Quitério, A., Costa, J., Martins, M., Martins, J., Onofre, M., Gerlach, E., Hermann, C. (2017). Educação física: Avaliação das competências motoras em alunos de seis anos, do primeiro ano de escolaridade. Retos, 31, 259-263

Rodero, C., Díaz, A., Guerrero, M. \& Duque, M. (2016). Análisis de la fiabilidad y validez de un cuestionario docente. Revisión, 9(1), 2.

Ruiz-Peìrez L, Barriopedro-Moro M, Ramoìn-Otero, M., Palomo-Nieto, M, Rioja-Collado N, Garciìa-Coll V. (2017). Evaluar la coordinación motriz global en educación secundaria: El test Sportcomp test. RICYDE: Revista Internacional de Ciencias Del Deporte, 11(49), 285-301. https://doi.org/10.5232/ricyd

Scheuer, C., Herrmann, C., \& Bund, A. (2019). Motor tests for primary school aged children: A systematic review. Journal of sports sciences, 37(10), 1097-1112.

Suárez, O. (2007). Aplicación del análisis factorial a la investigación de mercados. Caso de estudio. Scientia et technica, 1(35).

Wu, M., Lian, X., Lu, S., \& Wang, Z. (2017). Infant motor and cognitive abilities and subsequent executive function. Infant Behavior and Development, 49, 204-213.

Zueck, C., Alonso, A., García, R., Margarita, J., Villalobos, R., \& Eduardo, H. (2020). Satisfacción en las clases de Educación Física y la intencionalidad de ser activo en niños del nivel de primaria Satisfaction in the Physical Education classroom and intention to be physically active in Primary school children, 2041, 33-40.

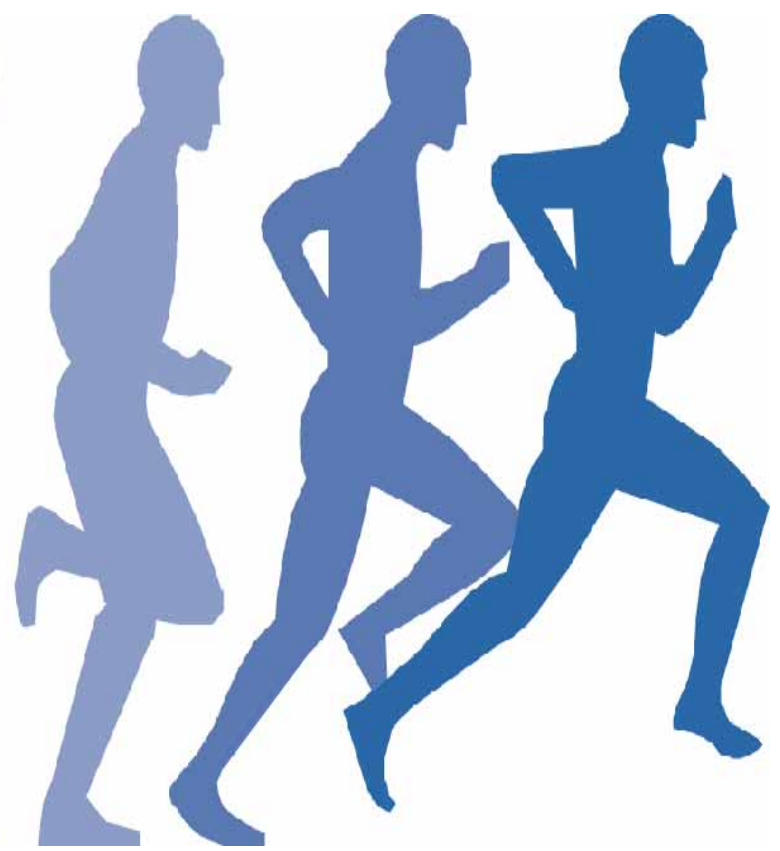

